Article

\title{
CA-170 - A Potent Small-Molecule PD-L1 Inhibitor or Not?
}

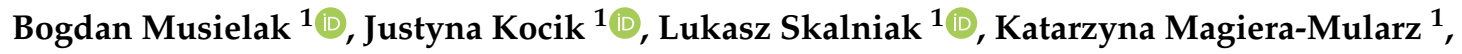 \\ Dominik Sala ${ }^{1}$, Miroslawa Czub ${ }^{1}$, Malgorzata Stec ${ }^{2}$, Maciej Siedlar ${ }^{2}$, Tad A. Holak ${ }^{1}$ and \\ Jacek Plewka 1,*(D) \\ 1 Faculty of Chemistry, Jagiellonian University, Gronostajowa 2, 30-387 Krakow, Poland \\ 2 Department of Clinical Immunology, Institute of Pediatrics, Jagiellonian University Medical College, \\ Wielicka 265, 30-663 Krakow, Poland \\ * Correspondence: jacek.plewka@uj.edu.pl; Tel.: +0048-12-686-2435
}

Received: 1 July 2019; Accepted: 30 July 2019; Published: 1 August 2019

\begin{abstract}
CA-170 is currently the only small-molecule modulator in clinical trials targeting PD-L1 and VISTA proteins - important negative checkpoint regulators of immune activation. The reported therapeutic results to some extent mimic those of FDA-approved monoclonal antibodies overcoming the limitations of the high production costs and adverse effects of the latter. However, no conclusive biophysical evidence proving the binding to hPD-L1 has ever been presented. Using well-known in vitro methods: NMR binding assay, HTRF and cell-based activation assays, we clearly show that there is no direct binding between CA-170 and PD-L1. To strengthen our reasoning, we performed control experiments on AUNP-12 - a 29-mer peptide, which is a precursor of CA-170. Positive controls consisted of the well-documented small-molecule PD-L1 inhibitors: BMS-1166 and peptide-57.
\end{abstract}

Keywords: CA-170; PD-1/PD-L1; NMR; HTRF; immune checkpoint

\section{Introduction}

Alongside chemo- and radiotherapy, surgery, and other "targeted treatments", cancer immunotherapy (called also immuno-oncology) is now regarded as the fifth pillar of cancer treatment, mainly due to a rapid development of potent immune checkpoint-blocking (ICB) therapeutic inhibitors [1-4]. These therapies unleash the native immune system by overcoming tumor-induced immunosuppression demonstrating impressive results. Among them, ICB agents, anti-PD-1/PD-L1 modulators have recently gained momentum and well-deserved acknowledgement in both academia (Nobel Prize in Physiology or Medicine in 2018 for James Allison and Tasuku Honjo for their discovery of cancer therapy by inhibition of negative immune regulation $[5,6]$ ) and pharmaceutical market ( $\$ 5$ billion in 2016 [7] and over 1500 different clinical studies on PD-1/PD-L1 agents as of 2017 (comprising mostly of combination therapies) [8]).

Programmed cell death protein 1 (known also as PD-1 and CD279) and its naturally occurring ligand PD-L1 (B7-H1, CD274) are transmembrane glycoprotein receptors characterized by $\beta$-sandwich immunoglobulin-like extracellular domains (seven $\beta$-strands organized in two sheets connected via a disulfide bridge) (Figure 1A) [9,10]. PD-1 is expressed at the cell surface of activated T and B cells, monocytes, dendritic cells and natural killer (NK) $\mathrm{T}$ cells [11,12]. Its extracellular $\mathrm{IgV}$ domain is followed by a transmembrane region and an intracellular tail, which contains two tyrosine-based immunoreceptor signaling motifs: the switch motif (ITSM), and the inhibitory motif (ITIM) [13]. Similarly, human PD-L1 (hPD-L1) also contains extracellular IgV domain, responsible for the binding to human PD-1 (hPD-1), followed by the IgC domain and a transmembrane domain. The fully human PD-1/PD-L1 complex was first structurally characterized in 2015 [9] and is reported to have the 1:1 
stoichiometry with partners interacting via the IgV domains by strands from the GFCC' beta sheets of both proteins placing them perpendicular to each other. The interface has a total surface area of $1970 \AA^{2}$ and is maintained by polar and hydrophobic interactions. More polar interactions comprising, hydrogen bonds and salt bridges, are observed at the periphery of the hydrophobic core based mainly on alkyl- $\pi$ and $\pi-\pi$ interactions. Interestingly, only PD-1 undergoes significant structural rearrangement upon binding, most notably in the $\mathrm{CC}^{\prime}$ loop, which transits from an open conformation in the apo form to a closed one in $C \beta$ strand [9].

A

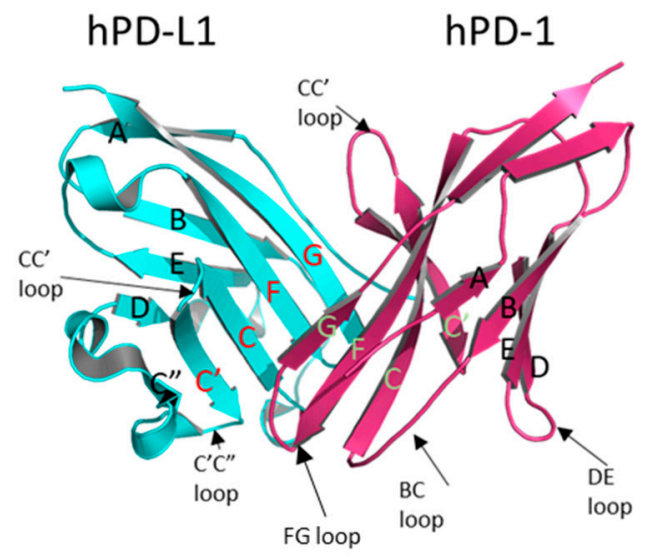

B

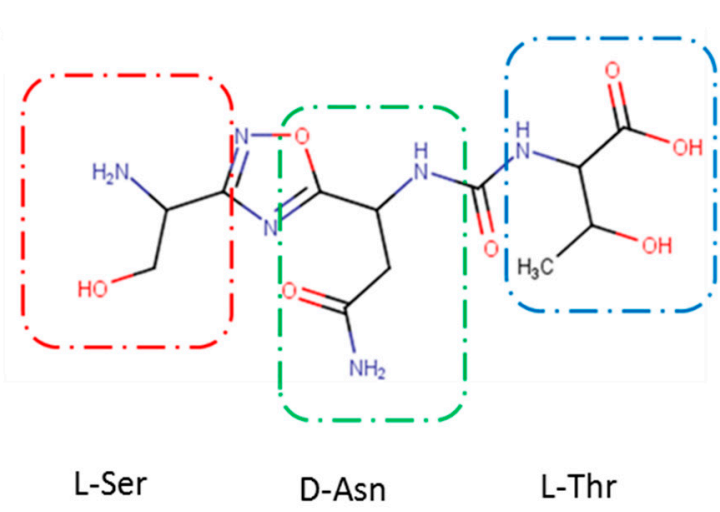

C SNTSESF-NH SNTSESFKFRVTQLAPKAQIKE-NH2

Figure 1. (A) Structure of the hPD-1/hPD-L1 complex. hPD-L1 in cyan, hPD-1 in purple; The strands of $\mathrm{IgV}$ are shown with names. Strands involved in the protein-protein interaction (PPI) are coded in red for hPD-L1 and green for hPD-1 (adapted from [9]). (B) The putative structure of CA-170 with building blocks indicated by boxes. (C) Structure of the AUNP-12 peptide composed of 4 hPD-1 parts: 2x BC loop (SNTSESF) in red connected via lysine to the D strand (sequence FRVTQ), purple; the FG loop (sequence LAPKA) in green, and the G strand (sequence QIKE), orange.

Physiologically, the PD-1/PD-L1 complex negatively regulates the immune response and provides so called T cell homeostasis [14,15]. Cancer cells often abuse this mechanism by overexpressing PD-L1 as a strategy to suppress anti-cancer activity of the immune system [16,17]. Therefore, disrupting the PD-1/PD-L1 complex at the cancer cell-T cell interface restores lymphocyte activity and has become an attractive target for pharmaceutical companies resulting in the development of potent monoclonal antibody-based therapies that have demonstrated remarkable therapeutic successes. Immunotherapies based on monoclonal antibodies (mAbs) are limited, though, by the properties of relatively big protein agents; namely the need for intravenous administration, immune-related adverse effects or high production costs [18-20]. Small molecule-based therapeutics are believed to serve as a remedy for those shortcomings, in most cases being orally bioavailable and cheaper in manufacturing, while presenting improved pharmacokinetics and with greater diffusion rates [21,22]. However, as of today, there are no FDA-approved small-molecule modulators for the PD-1/PD-L1. 
In the recent years, several attempts for the development of non-mAb PD-1/PD-L1 inhibitors, such as macrocyclic peptides, peptidomimetic molecules and nonpeptidic small molecules, were presented and disclosed in particular by Bristol-Myers Squibb (BMS) and Aurigene Discovery Technologies Limited (summarized in details in patent reviews [23-29]). In 2016, Aurigene in collaboration with Curis issued phase I trials of CA-170, a small-molecule inhibitor against PD-L1/L2 and VISTA, with low nanomolar potencies for the treatment of advanced solid tumors and lymphomas (NCT02812875, clinicaltrials.gov). CA-170 is also undergoing phase II clinical trials for lung cancer, head and neck/oral cavity cancer, MSI-H positive cancers and Hodgkin lymphoma in India (CTRI/2017/12/011026, ctri.nic.in). According to the preclinical in vitro and in vivo data reported by the company, CA-170 demonstrated dose-dependent enhancement in the proliferation of PD-L1, PD-L2, and VISTA-inhibited T lymphocytes, and exhibited antitumor effects similar to those of antibodies, including tumor shrinkages and prolonged stable disease without adverse effects [30-34].

Aurigene and Curis have not disclosed the structures of CA-170, however, based on the analysis of Aurigene patents and the fact that it is derived from the AUNP-12 peptide with known structure [35], CA-170 is most likely the compound 4 from the patent WO 2015/033301 A [36], being a peptidomimetic, composed of a L-serine, D-asparagine and L-threonine connected via diacylhydrazine and urea linker moieties, as presented in Figure 1 B. The same structures are advertised under the name CA-170 on following webpages of chemical vendors [37-40]. Its precursor, AUNP-12 peptide (Figure 1 C), was selected based on a rational structure-activity relationship (SAR) study, and presented a 100\% activity in the mouse splenocyte proliferation assay [41].

Despite being described as extremely potent with $\mathrm{EC}_{50}$ values of 17 and $0.72 \mathrm{nM}$ for CA-170 and AUNP-12 peptide, respectively [36,41], no biophysical data providing direct binding to any of the reported targets is available, which has already rose some concerns suggesting that these compounds may act on the broader PD-1/PD-L1 pathway rather than the mentioned proteins itself [23]. Here, we employed number of standard biophysical and biochemical methods, commonly used to assess the binding of tested molecules to the target proteins, in a conclusive way to determine affinities of CA-170, and its precursor AUNP-12, to hPD-L1. We also compare these molecules to the two well-characterized reference compounds - small-molecule BMS-1166 and the macrocyclic peptide-57 [42].

\section{Results}

\subsection{CA-170 Does Not Bind to hPD-L1 According to the NMR Binding Assay}

Nuclear magnetic resonance (NMR) is routinely employed to characterize small proteins, protein-protein and ligand-protein interactions [43]. Performing simple 2D experiment, such as heteronuclear multiple quantum coherence spectroscopy (HMQC) yields a two-dimensional map with a peak for each unique proton attached to heteroatom creating a kind of an individual fingerprint for each protein. Using this approach, one could verify if a given agent binds to the target protein by observing, whether the addition of tested agent induces changes of the initial protein spectrum [44]. The extent of those chemical shifts on the spectrum can be quantified to determine the affinity of tested molecule.

The affinities of CA-170 and AUNP-12 to hPD-L1 were assessed with the ${ }^{1} \mathrm{H}$ and ${ }^{1} \mathrm{H}-{ }^{15} \mathrm{~N}$ HMQC NMR spectroscopy. In the first experiment, the CA-170 compound was titrated against the ${ }^{15} \mathrm{~N}$-labeled hPD-1-binding single domain of hPD-L1 (amino acids 18-134) and against the entire extracellular domain of the hPD-L1 protein (residues 18-239). In both 1D and 2D NMR spectra, no changes were observed for signals of the protein of hPD-L1, even when the 10-fold excesses of compound was used (Figure 2A,B). These results indicate that CA-170 does not bind to hPD-L1 even at the elevated concentration as no peak shift or broadening of the NMR signals were observed. 

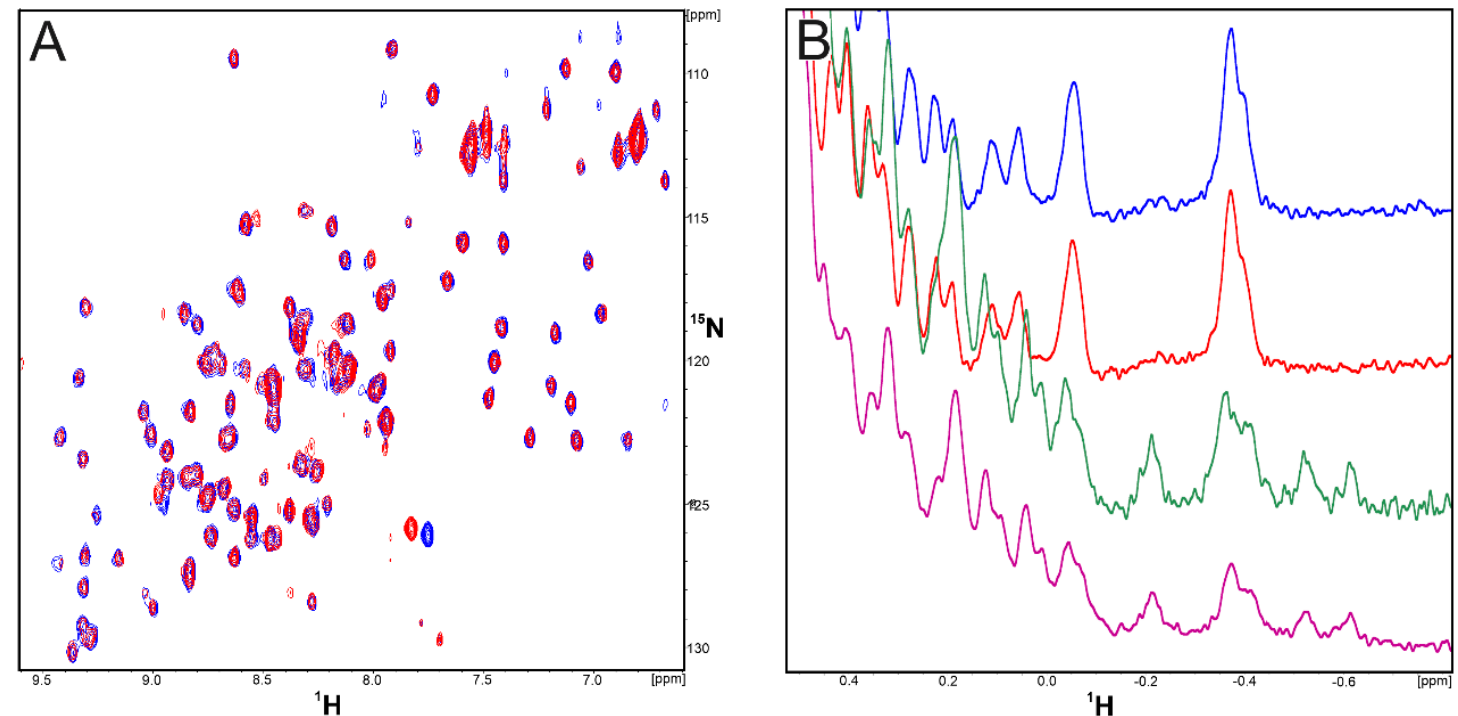

Figure 2. (A) ${ }^{1} \mathrm{H}^{15} \mathrm{~N}$ HMQC spectra of apo-hPD-L1 (18-134) (blue) and hPD-L1 (18-134) with CA-170 (red) in the molar ratio protein/CA-170 1:10, respectively. (B) ${ }^{1} \mathrm{H}$ NMR spectra of apo-hPD-L1 (18-134) (blue), hPD-L1 (18-134) with CA-170 (red) in the molar ration 1/10, apo-hPD-L1 (18-239) (purple), and hPD-L1 (18-239) with CA-170 (green) in the molar ration 1/10. In none of spectra any interactions of the tested compound with the hPD-L1 protein were observed as the recorded peaks overlay each other.

Although CA-170 is advertised as PD-L1 binder, we tested if it exhibits any interactions with hPD-1 to test all possibilities of disrupting the PD-1/PD-L1 complex. However, as in previous results, no interaction with hPD-1 protein was observed (Figure 3A,B). Since the selection method for the compound and in vitro assays were performed on the murine system showing spectacular results, we decided to conduct the same NMR binding experiment using mouse PD-L1 (mPD-L1) (Figure 3B). However, once again we did not observe any indication of the binding between the CA-170 compound and mPD-L1.
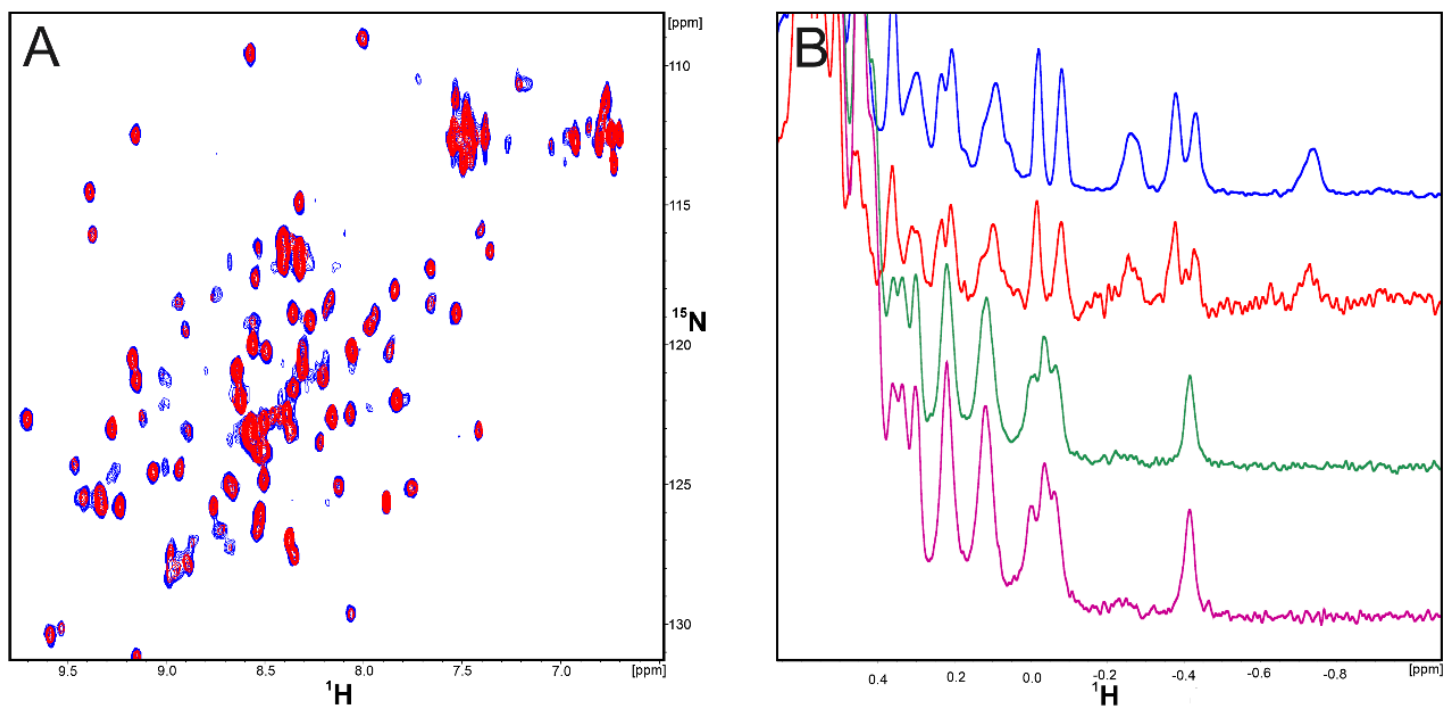

Figure 3. (A) ${ }^{1} \mathrm{H}-{ }^{15} \mathrm{~N}$ HMQC spectra of apo-hPD-1 (blue) and hPD-1 with CA-170 (red) in the molar ration 1/10. (B) ${ }^{1} \mathrm{H}$ NMR spectra of apo- hPD-1 (blue), hPD-1 with CA-170 (red) in the molar ratio 1/10, apo-mPD-L1 (green), and mPD-L1 with CA-170 (purple) in the molar ratio 1/10.

Since CA-170 was derived from the AUNP-12 peptide, which is composed of the 4 fragments of hPD-1, we decided to also perform the NMR binding assay to test the affinity of AUNP-12 to 
hPD-L1 in the same way. The analysis of perturbations of chemical shifts of protein NMR signals in the HMQC spectra of ${ }^{15} \mathrm{~N}$ hPD-L1 allowed us to determine that the AUNP-12 binds to the short construct of hPD-L1 (18-134) with the $\mathrm{K}_{\mathrm{D}}$ at the millimolar level indicated by slight chemical shift perturbations (Figure S1A). Unambiguous changes in the NMR spectra are observed for short hPD-L1 with the excess of AUNP-12 at the molar ratio of 1/5. The rest of the results of NMR experiments for AUNP-12 with hPD-L1 (18-239) and with hPD-1 showed no interaction of the peptide and the proteins (Figures S1B and S2A,B).

Therefore, we determined in a conclusive way that neither CA-170 nor AUNP-12 binds to human or mouse PD-L1 or human PD-1. As the reference positive controls for the NMR binding assay, we show the NMR spectra of the well-known hPD-L1 binders: macrocyclic peptide-57 and small-molecule BMS-1166 developed by Bristol-Myers Squibb, which bind strongly to hPD-L1 at equimolar concentrations (Figure S3) (Adapted from Skalniak et al., Oncotarget, 2017 [42]).

\subsection{CA-170 Cannot Disrupt hPD-1/hPD-L1 Complex as Determined with HTRF Assay}

Regardless of the NMR measurements, we performed the $\mathrm{IC}_{50}$ determination for CA-170 and AUNP-12 peptide with the hPD-1/hPD-L1 complex using Homogenous Time Resolved FRET (HTRF), which is currently a standard methodology to determine potency of the inhibitors in numerous publications and patents, due to its robustness and absence of false-positive results [45]. We used increasing concertation of the inhibitors against standardized $5 \mathrm{nM}$ of hPD-L1 and $50 \mathrm{nM}$ of hPD-1 in duplicates. The resulting normalized data was then fitted with the Hill's equation. We also determined $\mathrm{IC}_{50}$ of well-known hPD-L1 inhibitors as the positive reference: BMS-1166 and peptide-57 to cross-validate the results with the literature (Figure 4).

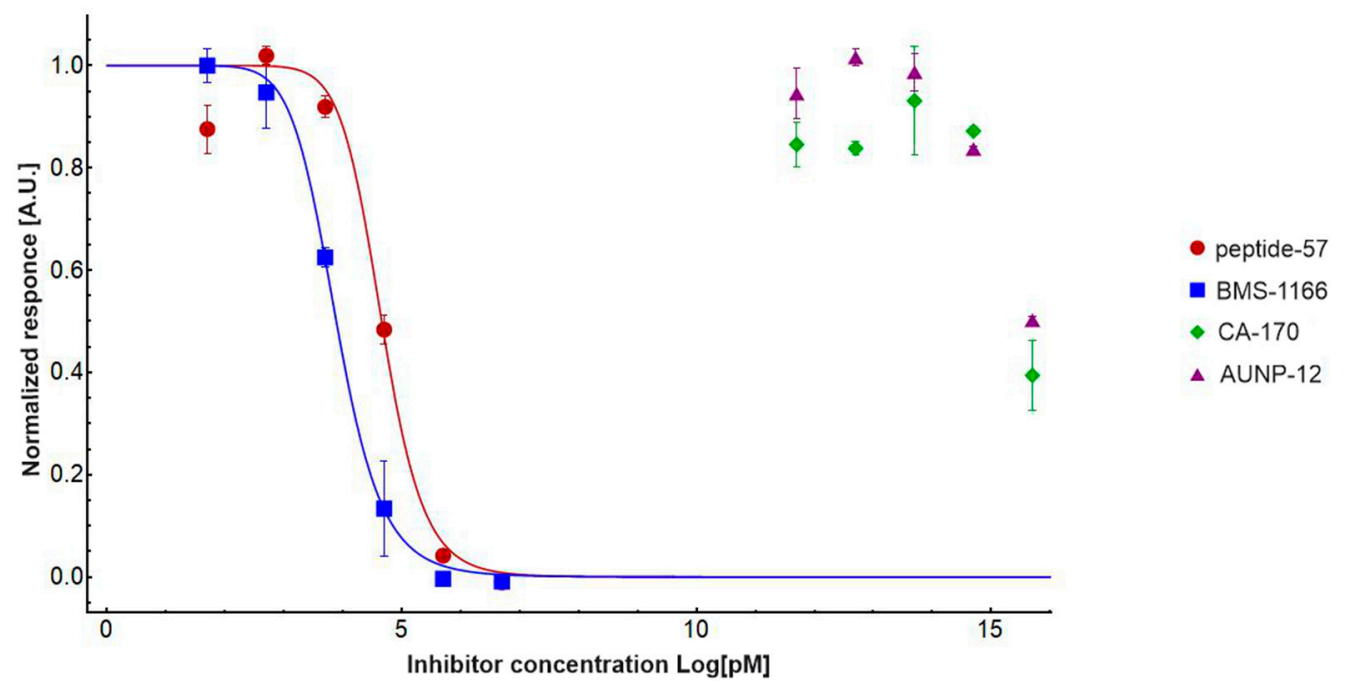

Figure 4. Determination of the potency for reference compounds peptide-57 (red circles), BMS-1166 (blue squares) as compared to almost no response from CA-170 (green diamonds), and AUNP-12 (violet triangles). Fitting was performed using normalized Hill's equation.

From the above consideration, it is clear that both reference compounds are potent in dissociating the hPD-1/hPD-L1 complex with $\mathrm{IC}_{50}$ of $45.4 \pm 0.001 \mathrm{nM}$ and $7.7 \pm 0.01 \mathrm{nM}$ for peptide-57 and BMS-1166, respectively, which is in hand with the values reported in the literature [22,42]. However, both AUNP-12 and CA-170 only marginally dissociated the complex at the highest used concentration of $5 \mathrm{mM}$ indicating that the estimated $\mathrm{IC}_{50}$ would be above $5-10 \mathrm{mM}$. It should be noted, though, that at such high concentrations the interactions of small molecules with isolated protein complex may be unspecific. Therefore, we presented in a conclusive way that using standard biophysical methods for assessing affinities of a small compound towards target proteins by NMR and HTRF assays, neither AUNP-12 nor CA-170 exhibits potency in dissociation of the hPD-1/hPD-L1 complex. 


\subsection{CA-170 Fails to Restore the Activation of hPD-1/hPD-L1-Blocked Effector Jurkat T Cells}

To verify the activity of compound CA-170 in a hPD-1/hPD-L1-sensitive cell line test, the compound was challenged in the immune checkpoint blockade bioassay [46]. For this, antigen-presenting CHO-K1 cells overexpressing hPD-L1 and T Cell Receptor (TCR) Activator (hPD-L1 aAPCs) were co-cultured with the effector Jurkat T cells overexpressing hPD-1 and a luciferase gene controlled by the NFAT-Response Element (hPD-1 Effector Cells, hPD-1 ECs). In the assay, the activation of TCR-signaling by the TCR-activator is inhibited by the hPD-1/hPD-L1 interaction. Upon hPD-1/hPD-L1 blockade, normal Jurkat $\mathrm{T}$ cell activation is restored, as monitored by the increased luciferase activity. In order to ensure that our CHO-K1 model cells (hPD-L1 aAPCs) are suitable for testing PD-L1-binding compounds, we have verified the expression of human PD-L1 protein in these cells. Western blot analysis confirmed that aAPCs express human PD-L1, while original CHO-K1 cells and CHO-K1 cells transfected with the TCR Activator-coding vector but not the human PD-L1-encoding vector did not show any expression of this protein (Figure $5 \mathrm{~A}$ ). Moreover, the human PD-L1 protein is successfully exposed on the surface of aAPCs, as confirmed with the flow cytometry analysis (Figure $5 \mathrm{~B}$ ).

A

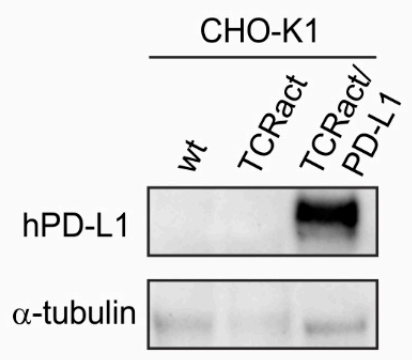

B

human PD-L1 expression on the hPD-L1 aAPCs

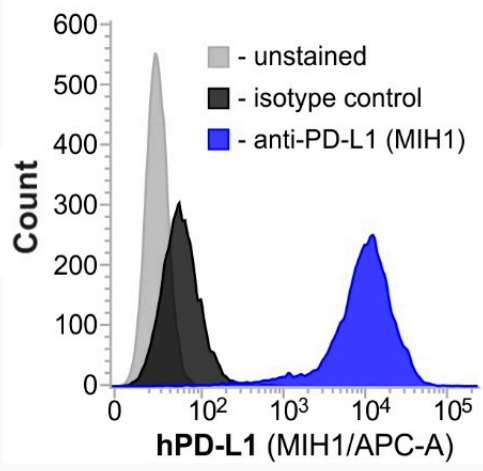

C

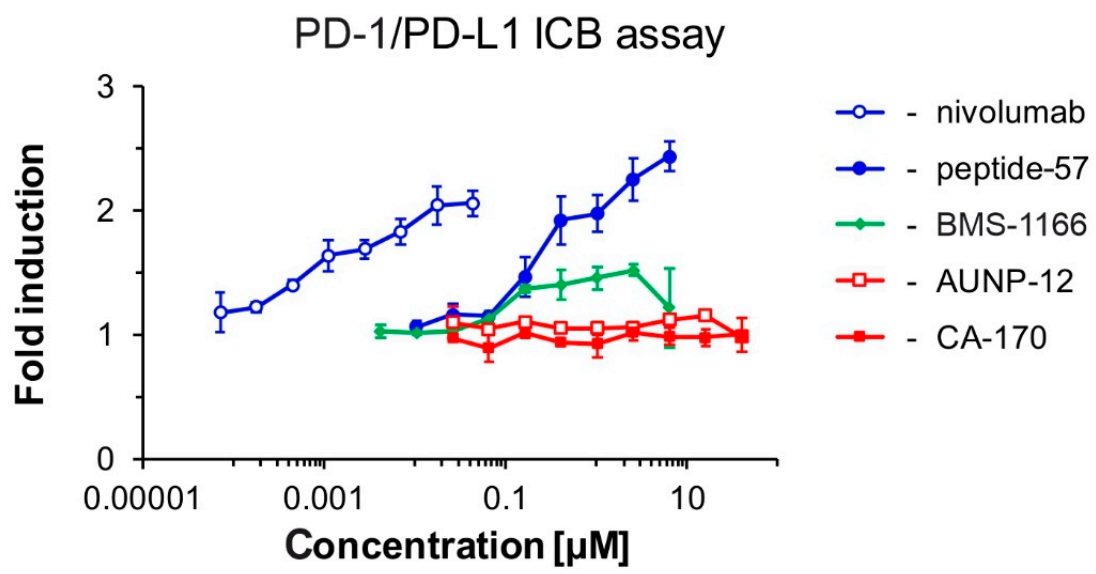

Figure 5. The activities of the tested molecules in the cell-based in vitro immune checkpoint blockade assay. (A) Western blot analysis of the expression of the human PD-L1 protein in the hPD-L1 aAPCs (artificial antigen-presenting cells, CHO-K1 TCRact/PD-L1) in comparison to the original $\mathrm{CHO}-\mathrm{K} 1$ cells $(\mathrm{CHO}-\mathrm{K} 1 \mathrm{wt})$ and $\mathrm{CHO}-\mathrm{K} 1$ overexpressing only the TCR Activator molecule (CHO-K1 TCRact). (B) Flow cytometry analysis of the expression of human PD-L1 on the surface of the hPD-L1 aAPCs. (C) Comparison of bioactivities of the known PD-L1-targeting molecules: nivolumab, peptide-57, BMS-1166, and the tested molecules, CA-170 and AUNP-12 in the hPD-1/hPD-L1 immune checkpoint assay. 
In the immune checkpoint blockade assay, three positive control molecules were used: small-molecule BMS-1166, a macrocyclic peptide-57, and an approved monoclonal antibody nivolumab [47]. All three compounds restored the activation of hPD-1 ECs, however significant differences in their potencies were noticed (Figure $5 \mathrm{C}$ ). According to the assay, the $\mathrm{EC}_{50}$ for nivolumab was $1.4 \mathrm{nM}$, for BMS-1166 - $83.4 \mathrm{nM}$, and for peptide-57 - $185.5 \mathrm{nM}$ as determined from Hill's fitting, which are close to the values reported in the HTRF assay. This observation is also in agreement with our previous reports, where we have shown that the macrocyclic peptides and BMS compounds, although active, present much higher $\mathrm{EC}_{50}$ values and lower maximal effects compared to therapeutic antibodies [42,47]. On the other hand, neither CA-170, nor its precursor AUNP-12, was able to provide the activation of Jurkat T cells, repressed by the hPD-1/hPD-L1 checkpoint, up to the concentration of $40 \mu \mathrm{M}$ of the compounds. This is a strong evidence that the compounds fail to interfere specifically with the hPD-1/hPD-L1 immune checkpoint in the biological system.

\section{Discussion}

Small-molecule modulators for the hPD-1/hPD-L1 pathway are of a great interest to both patients and pharmaceutical companies. This is reflected by the increasing number of patent applications for small-molecular scaffolds. HTRF or AlphaScreen assay are used as standardized methods to determine the potency of screen compounds in disrupting the hPD-1/hPD-L1 complex. However, even though many of them exhibits sub-nanomolar affinities, none of them progressed to the clinical trials, due to multiple reasons, such as poor drugability, toxicity profile of the compounds, etc. The only compound currently in clinical trial phase I is CA-170, which is advertised as the modulator that dually targets VISTA and hPD-L1. The structure of CA-170 has not been formally disclosed. Based on the patent and review analysis, we have concluded that it is the compound 4 from the patent WO 2015/033301 A [36]. Moreover, Aurigene/Curis did not provide any biophysical results confirming direct binding of their compound to any of the advertised targets, neither for CA-170 nor to its precursor AUNP-12, raising some speculations regarding the mechanism of the action of these modulators. The only confirmation of the potency of CA-170 and AUNP-12 comes from the proliferation restoration assays and IFN- $\gamma$ secretion rescue from mouse splenocyte and human PBMC. Lack of orthogonal methods for the confirmation of the potency and inconclusive nature of applied methodology made us question the mechanism of action for CA-170 and AUNP-12. Therefore, we conducted our own investigation to determine the binding affinities of CA-170 and AUNP-12 to hPD-L1 in a conclusive way using well acknowledged methods of NMR binding assay, HTRF and the hPD-1/hPD-L1 immune checkpoint blocking assay.

Based on the above consideration we state that neither CA-170 nor its precursor AUNP-12 exhibit any binding to hPD-L1 that would be strong enough to disrupt the hPD-1/hPD-L1 complex. Furthermore, they do not bind to mouse PD-L1 (mPD-L1) or hPD-1 either. Lack of the activity in the precursor peptide does not rule out the possibility that its fragments alone may be potent. Moreover, we do not exclude the possibility that this family of compounds may act downstream from the hPD-1 receptor or on any other $\mathrm{T}$ cell-activating pathway, since the compounds clearly present promising therapeutic results according to the data provided by Aurigene and Curis [48].

We hypothesize that the reason why Aurigene compounds do not bind to hPD-L1 may be explained by the conceptual fault in the initial "rational design". A design starting point, the compound AUNP-12, is a hybrid of the peptide sequences extracted from hPD-1, that are supposed to be close to its interaction surface with hPD-L1. However, if we look at the locations of particular peptide fragments of AUNP-12 (Figure 6), it shows that only the LAPKAQIKE sequence is present at the direct interface of the hPD-1/hPD-L1 interaction. This might be the reason why AUNP-12 presents some, albeit weak, binding to hPD-L1 in the NMR assay at high compound concentrations. However, the BC loop containing the SNT motif, that constitutes the optimized CA-170 compound, is pointing away from the PD-1/PD-L1 interface as can be inferred from the fully human PD-1/PD-L1 complex structure first reported by Zak et al. in 2015 [9]. This explains the lack of direct binding between 
hPD-L1 and CA-170, which does not justify the rationality of the selection of this particular part of hPD-1 as a putative inhibitor.

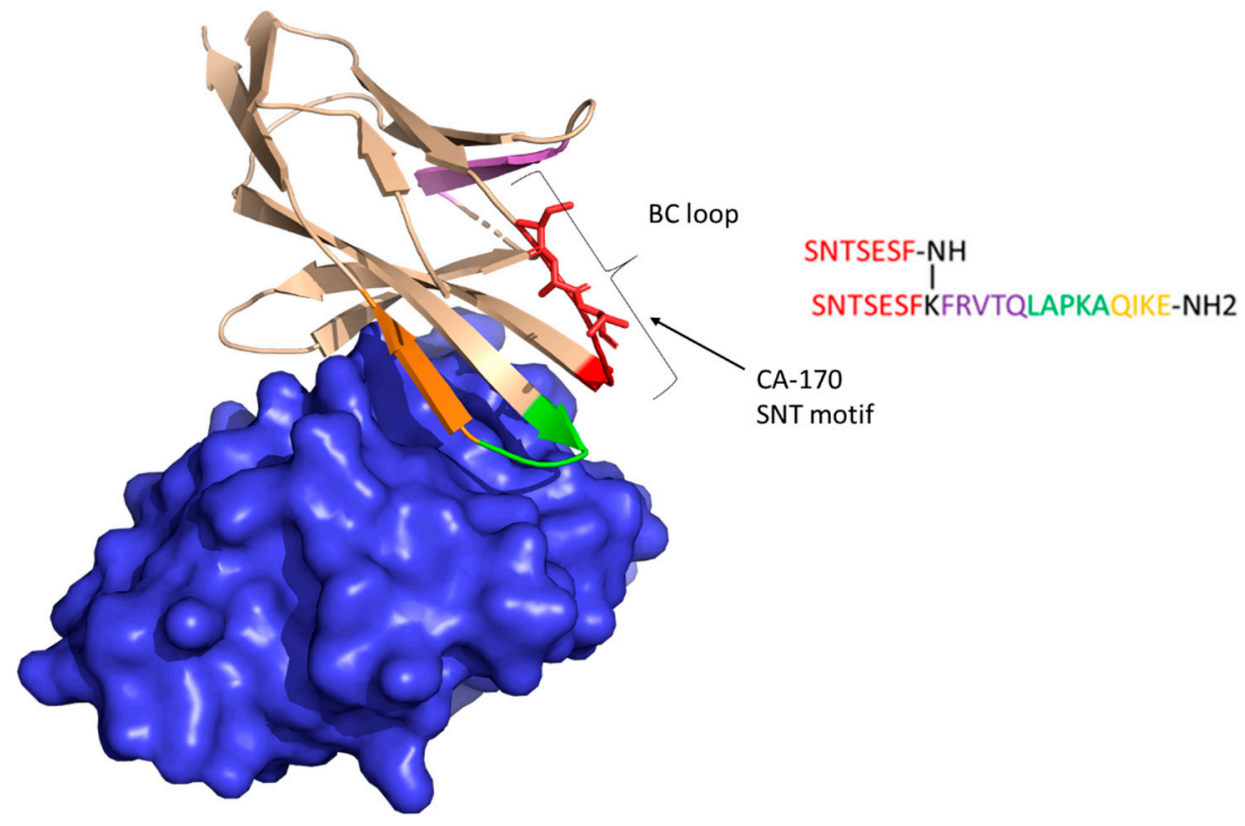

Figure 6. hPD-1 (light brown cartoon) and hPD-L1 (blue surface) in complex from 4ZQK PDB. The BC loop is highlighted. The AUNP-12 peptide sequence (on the right) is color coded on corresponding strands of hPD-1. SNT-motif from CA-170 is represented as sticks in BC loop.

Therefore, both AUNP-12 and CA-170 are not targeted at hPD-L1 as advertised, which may still be in agreement with the results from the splenocyte screening assay, given that the assay does not have to be hPD-1/hPD-L1 specific due to its complex nature. Despite above consideration, CA-170 shows promising results in clinical trials and further investigation of the mode of action, especially identifying the binding partner/s, is highly recommended.

\section{Materials and Methods}

\subsection{Materials}

CA-170 was purchased from MedChemExpress (Monmouth Junction, NJ, USA) Cas: 1673534-76-3, AUNP-12 was purchased from SelleckChem (Houston, TX, USA) BMS-1166 was synthesized according to the protocol from described in [22], peptide-57 was synthesized as described in [49].

\subsection{Protein Expression and Purification}

The gene encoding hPD-1 (amino acids 33-150, Cysteine 93 mutated to serine) was cloned into pET-24d, the gene encoding hPD-L1 (amino acids 18-134 with C-terminal HisTag) was cloned into pET-21b, the genes encoding hPD-L1 (amino acids 18-239 with C-terminal HisTag) and mPD-L1 (amino acids 38-134) was cloned into pET-28a, respectively. Proteins were expressed in the Escherichia coli BL21 (DE3). Bacterial cells were cultured at $37^{\circ} \mathrm{C}$ in LB or M9 minimal medium containing ${ }^{15} \mathrm{NH}_{4} \mathrm{Cl}$ as the sole nitrogen source to achieve ${ }^{15} \mathrm{~N}$ isotope labeling. Proteins expression was induced with $1 \mathrm{mM}$ Isopropyl $\beta$-D-1-thiogalactopyranosid (IPTG) at $\mathrm{OD}_{600}$ of 0.8 and the cells were cultured overnight. For hPD-1, hPD-L1 and mPD-L1 temperature was lowered to $28^{\circ} \mathrm{C}$, for hPD-L1(18-239) temperature was left at $37^{\circ} \mathrm{C}$. The inclusion bodies purification was carried out as described previously [9]. Afterwards proteins were refolded by drop-wise dilution into solution containing $0.1 \mathrm{M}$ Tris $\mathrm{pH} 8.0,0.4 \mathrm{M} \mathrm{L}$-Arginine hydrochloride, $2 \mathrm{mM}$ EDTA, $5 \mathrm{mM}$ cystamine and $0.5 \mathrm{mM}$ cysteamine for hPD-1 and $0.1 \mathrm{M}$ Tris $\mathrm{pH}$ 8.0,

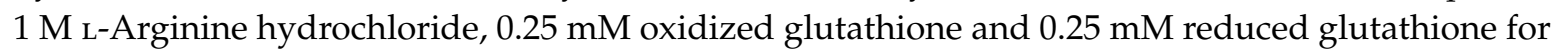


hPD-L1, human PD-L1(18-239) and mPD-L1. After refolding, proteins were dialyzed 3 times against solution containing $10 \mathrm{mM}$ Tris $\mathrm{pH} 8.0$ and $20 \mathrm{mM} \mathrm{NaCl}$. Finally, proteins were purified by SEC (size-exclusion chromatography) on HiLoad 26/600 Superdex 75 column (GE Healthcare, Chicago, IL, USA) in 25mM sodium phosphate $\mathrm{pH} 6.4$ with $100 \mathrm{mM} \mathrm{NaCl}$ for hPD-1 or in PBS pH 7.4 for hPD-L1, hPD-L1(18-239) and mPD-L1.

\subsection{NMR Binding Assay}

For NMR measurements, the buffer was exchanged by gel filtration to PBS pH 7.4. 10\% $(v / v)$ of $\mathrm{D}_{2} \mathrm{O}$ was added to the samples to provide the lock signal. All spectra were recorded at $300 \mathrm{~K}$ using a Bruker Avance III $600 \mathrm{MHz}$ spectrometer. Binding of the compounds was analyzed by titrating the ${ }^{15} \mathrm{~N}$-labeled hPD-L1/hPD-1 and recording the ${ }^{1} \mathrm{H}$ and ${ }^{1} \mathrm{H}-{ }^{15} \mathrm{~N}$ HMQC spectra prior to and after the addition of the compounds.

\subsection{Homogenous Time Resolved FRET}

HTRF assay was performed using the certified Cis-Bio assay kit at $20 \mu \mathrm{L}$ final volume using their standard protocol ( $5 \mathrm{nM}$ of h-L1 and $50 \mathrm{nM}$ of hPD-1 in the final formulation). To determine the half maximal inhibitory concentration $\left(\mathrm{IC}_{50}\right)$ of tested compounds, measurements were performed on individual dilution series. After mixing all components according to Cis-Bio protocol, the plate was left for $2 \mathrm{~h}$ incubation at room temperature followed by TR-FRET measurement on Tecan Spark 20M. Collected data was background subtracted on the negative control, normalized on the positive control, averaged and fitted with normalized Hill's equation to determine the $\mathrm{IC}_{50}$ value using Mathematica 12.

\subsection{Cell Culture}

CHO K-1 cells overexpressing hPD-L1 and the recombinant TCR ligand (hPD-L1 Antigen Presenting Cells, hPD-L1 aAPCs) (Promega, Madison, WI, USA) and Jurkat T cells overexpressing hPD-1 and carrying a luciferase reporter gene under the control of Nuclear Factor of Activated T-cells Response Element (NFAT-RE) (hPD-1 Effector Cells, hPD-1 ECs, Promega) were cultured in RPMI-1640 medium (Biowest, Billerica, MA, USA) supplemented with 10\% Fetal Bovine Serum (FBS, Biowest) and $200 \mathrm{mM}$ L- Glutamine (Biowest) in the presence of G418 (250 $\mu \mathrm{g} / \mathrm{mL}$, InvivoGen, San Diego, CA, USA) and Hygromycin B Gold (50 $\mathrm{g} / \mathrm{mL}$, InvivoGen) as selection antibiotics. The overexpression of hPD-L1 and TCR ligand in aAPCs and PD-1 in ECs were confirmed by flow cytometry and western blot analysis, respectively. PCR tests for Mycoplasma sp. contamination [50] were routinely performed and indicated negative results for both cell lines.

\section{6. hPD-1/hPD-L1 Immune Checkpoint Blockade Assay}

The activity of the inhibitors of hPD-1/hPD-L1 immune checkpoint was examined using the hPD-1/hPD-L1 Blockade Bioassay (Promega), according to the manufacturer's instructions. hPD-L1 aAPCs were seeded on 96-well (white) plates at the density 10000 cells/well $17 \mathrm{~h}$ prior to the experiment. The 2.5-fold dilutions of the small molecules or peptide-57 were first prepared in DMSO. On the day of the assay the compounds were diluted 1000-fold in the assay buffer (99\% RPMI 1640, 1\% FBS) to maintain the constant concentration of DMSO $(0.1 \%$ of total volume). The 2.5 -fold dilutions of nivolumab, a positive control anti-hPD-1 monoclonal antibody (Opdivo, Bristol-Myers Squibb, New York, NY, USA), were prepared in the assay buffer on the day of the assay. The culture medium was discarded from the wells and serial dilutions of either the small-molecule or antibody was added. Afterwards, Jurkat hPD-1 cells were seeded at the density of 20,000 cells per well in the assay's plates. After $6 \mathrm{~h}$ of the incubation in standard culture conditions, assay plates were equilibrated at ambient temperature for $10 \mathrm{~min}$, followed by a $20 \mathrm{~min}$ incubation with the Bio-GloTM Assay reagent (Promega). The luminescence was detected using the Infinite M200 reader. Half maximal effective concentrations ( $\mathrm{EC}_{50}$ values) were calculated from the Hill's curve fitting to the experimental data. 


\subsection{Western Blot Analysis}

Total cell lysates were prepared with RIPA buffer (Sigma Aldrich, Saint Louis, MO, USA) containing protease inhibitor cocktail (Sigma Aldrich). Following the electrophoresis and transfer to PVDF membranes, the membranes were blocked with 4\% BSA (BioShop, Burlington, Canada) in TBS-N buffer and incubated with primary antibody solution at $4{ }^{\circ} \mathrm{C}$ overnight. After that, four washes with TBS-N buffer, the incubation with secondary antibody for 1 h at room temperature, and additional four washes were done. Then, the detection was performed with the use of Clarity Western ECL Substrate (BioRad, Hercules, CA, USA) and ChemiDoc MP system (BioRad).

The following antibodies and dilutions were used: rabbit monoclonal anti-hPD-L1 (clone E1L3N, 1:1 000, Cell Signaling Technology (CST, Danvers, MA, USA), cat. 13684), rabbit monoclonal anti- $\alpha$-Tubulin (1:2000, CST, cat. 2125), goat peroxidase-conjugated anti-rabbit (1:3000, CST, cat. 7074).

\subsection{Flow Cytometry Analysis}

For the analysis of the expression of human PD-L1 on the surface of aAPC cells flow cytometry analysis was performed. The cells were seeded on 6-well plates and cultured till sub-confluency. The cells were detached from the plates with TrypLe Select Enzyme (Thermo Fisher Scientific, Waltham, MA, USA), placed on ice, washed 2 times with Flow Cytometry Staining Buffer (eBioscience, Waltham, MA, USA) and stained with mouse anti-human PD-L1 antibody (clone MIH1, conjugated with APC, cat. 17-5983). 17-4714 antibody was used as an isotype control of the staining. The cells were analyzed with the FACSCanto II cytometer.

\section{Conclusions}

CA-170 is the first small-molecule inhibitor targeted at PD-L1 that was introduced to phase I and II clinical trials. We, however, unambiguously determined that there is in fact no direct binding between CA-170 and its peptidic precursor AUNP-12 to PD-L1. In the NMR binding assay, neither of these molecules showed any interactions with the one- or two-domain PD-L1 in the human and mice variants as well as human PD-1. CA-170 and AUNP-12 were also not able to dissociate complex formation in the isolated system of the HTRF assay and cell-based assay mimicking in vivo conditions. All measurements were cross-validated with positive controls: peptide-57 and small-molecule BMS-1166. Therefore, proposed mechanism of the direct blocking of hPD-1/hPD-L1 interactions with CA-170 has to be changed.

Supplementary Materials: The following are available online at http://www.mdpi.com/1420-3049/24/15/2804/s1, Figure S1. (A) ${ }^{1} \mathrm{H}_{-}{ }^{15} \mathrm{~N}$ HMQC spectra of apo-hPD-L1 (18-134) (blue) and hPD-L1 (18-134) with AUNP-12 (red) in the molar ration 1/5. (B) ${ }^{1} \mathrm{H}$ NMR spectra of apo-hPD-L1 (18-134) (blue), hPD-L1 (18-134) with AUNP-12 (red) in the molar ration 1/5, apo-hPD-L1 (18-239) (purple), and hPD-L1 (18-239) with AUNP-12 (green) in the molar ration 1/5, Figure S2. (A) ${ }^{1} \mathrm{H}_{-}{ }^{15} \mathrm{~N}$ HMQC spectra of apo-hPD-1 (blue) and hPD-1 with AUNP-12 (red) in the molar ration 1/5. (B) ${ }^{1} \mathrm{H}$ NMR spectra of apo- hPD-1 (blue), hPD-1 with AUNP-12 (red) in the molar ration 1/5., Figure S3. ${ }^{1} \mathrm{H}$ NMR spectra of apo-hPD-L1 (18-134) (blue) and with BMS-1166 compound (green) and peptide-57 (red) in molar ration $1 / 1$, respectively.

Author Contributions: J.P. wrote the draft of the manuscript. All authors discussed the experiments and commented on the manuscript. B.M. performed NMR experiments; J.P. and M.C. performed HTRF; L.S. and J.K. carried out the cell-based assays; K.M.M. and D.S. provided support with preparation of expression plasmids and with protein purification. M.S. (Malgorzata Stec), M.S. (Maciej Siedlar), performed flow cytometry experiment and analysis, T.A.H. provided the expertise for data analysis and proofreading of the manuscript.

Funding: This research was partially funded (to T.A.H.) by the project POIR.04.04.00-00-420F/17-00 which is carried out within the TEAM programme of the Foundation for Polish Science co-financed by the European Union under the European Regional Development Fund. We acknowledge the MCB Structural Biology Core Facility (supported by the TEAM TECH CORE FACILITY/2017-4/6 grant from the Foundation for Polish Science) for valuable support.

Acknowledgments: J.K. acknowledges the support of InterDokMed project no. POWR.03.02.00-00-I013/16. K.M.M. is a recipient of the START fellowship from the Foundation for Polish Science (FNP). 
Conflicts of Interest: The authors declare no conflict of interest.

\section{References}

1. Pardoll, D.M. The blockade of immune checkpoints in cancer immunotherapy. Nat. Rev. Cancer 2012, 12, 252-264. [CrossRef] [PubMed]

2. Kelly, P.N. The Cancer Immunotherapy Revolution. Science 2018, 359, 1344-1345. [CrossRef] [PubMed]

3. Ribas, A.; Wolchok, J.D. Cancer immunotherapy using checkpoint blockade. Science 2018, 359, $1350-1355$. [CrossRef] [PubMed]

4. Dömling, A.; Holak, T.A. Programmed Death-1: Therapeutic Success after More than 100 Years of Cancer Immunotherapy. Angew. Chem. Int. Ed. 2014, 53, 2286-2288. [CrossRef] [PubMed]

5. Ledford, H.; Else, H.; Warren, M. Cancer immunologists scoop medicine Nobel prize. Nature 2018, 562, $20-21$. [CrossRef] [PubMed]

6. Kaiser, J.; Couzin-Frankel, J. Cancer immunotherapy sweeps Nobel for medicine. Science 2018, $362,13$. [CrossRef] [PubMed]

7. Global \$4.92 Billion Programmed Death-1 (PD-1) \& amp; Programmed Death Ligand-1 (PD-L1) Inhibitors Pipeline Analysis 2017-2025-Research and Markets. Available online: https://www.prnewswire.com/news-releases/global492-billion-programmed-death-1-pd-1--programmed-death-ligand-1-pd-11-inhibitors-pipeline-analysis-20172025---research-and-markets-300422553.html (accessed on 4 June 2019).

8. Tang, J.; Shalabi, A.; Hubbard-Lucey, V.M. Comprehensive analysis of the clinical immuno-oncology landscape. Ann. Oncol. 2018, 29, 84-91. [CrossRef]

9. Zak, K.M.; Kitel, R.; Przetocka, S.; Golik, P.; Guzik, K.; Musielak, B.; Dömling, A.; Dubin, G.; Holak, T.A. Structure of the Complex of Human Programmed Death 1, PD-1, and Its Ligand PD-L1. Structure 2015, 23, 2341-2348. [CrossRef]

10. Lin, D.Y.-w.; Tanaka, Y.; Iwasaki, M.; Gittis, A.G.; Su, H.P.; Mikami, B.; Okazaki, T.; Honjo, T.; Minato, N.; Garboczi, D.N. The PD-1/PD-L1 complex resembles the antigen-binding Fv domains of antibodies and T cell receptors. Proc. Natl. Acad. Sci. USA 2008, 105, 3011-3016. [CrossRef]

11. Chen, L.; Flies, D.B. Molecular mechanisms of T cell co-stimulation and co-inhibition. Nat. Rev. Immunol. 2013, 13, 227-242. [CrossRef]

12. Riella, L.V.; Paterson, A.M.; Sharpe, A.H.; Chandraker, A. Role of the PD-1 Pathway in the Immune Response. Am. J. Transplant. 2012, 12, 2575-2587. [CrossRef] [PubMed]

13. Chemnitz, J.M.; Parry, R.V.; Nichols, K.E.; June, C.H.; Riley, J.L. SHP-1 and SHP-2 associate with immunoreceptor tyrosine-based switch motif of programmed death 1 upon primary human $\mathrm{T}$ cell stimulation, but only receptor ligation prevents T cell activation. J. Immunol. 2004, 173, 945-954. [CrossRef] [PubMed]

14. Francisco, L.M.; Sage, P.T.; Sharpe, A.H. The PD-1 pathway in tolerance and autoimmunity. Immunol. Rev. 2010, 236, 219-242. [CrossRef] [PubMed]

15. Tumeh, P.C.; Harview, C.L.; Yearley, J.H.; Shintaku, I.P.; Taylor, E.J.M.; Robert, L.; Chmielowski, B.; Spasic, M.; Henry, G.; Ciobanu, V.; et al. PD-1 blockade induces responses by inhibiting adaptive immune resistance. Nature 2014, 515, 568-571. [CrossRef] [PubMed]

16. Ahmadzadeh, M.; Johnson, L.A.; Heemskerk, B.; Wunderlich, J.R.; Dudley, M.E.; White, D.E.; Rosenberg, S.A. Tumor antigen-specific CD8 T cells infiltrating the tumor express high levels of PD-1 and are functionally impaired. Blood 2009, 114, 1537-1544. [CrossRef] [PubMed]

17. Hawkes, E.A.; Grigg, A.; Chong, G. Programmed cell death-1 inhibition in lymphoma. Lancet Oncol. 2015, 16, e234-e245. [CrossRef]

18. Farid, S.S. Process economics of industrial monoclonal antibody manufacture. J. Chromatogr. B 2007, 848, 8-18. [CrossRef] [PubMed]

19. Baldo, B. Adverse events to monoclonal antibodies used for cancer therapy: Focus on hypersensitivity responses. Oncoimmunology 2013, 2, e26333. [CrossRef] [PubMed]

20. Michot, J.M.; Bigenwald, C.; Champiat, S.; Collins, M.; Carbonnel, F.; Postel-Vinay, S.; Berdelou, A.; Varga, A.; Bahleda, R.; Hollebecque, A.; et al. Immune-related adverse events with immune checkpoint blockade: A comprehensive review. Eur. J. Cancer 2016, 54, 139-148. [CrossRef]

21. Adams, J.L.; Smothers, J.; Srinivasan, R.; Hoos, A. Big opportunities for small molecules in immuno-oncology. Nat. Rev. Drug Discov. 2015, 14, 603-622. [CrossRef] 
22. Guzik, K.; Zak, K.M.; Grudnik, P.; Magiera, K.; Musielak, B.; Törner, R.; Skalniak, L.; Dömling, A.; Dubin, G.; Holak, T.A. Small-Molecule Inhibitors of the Programmed Cell Death-1/Programmed Death-Ligand 1 (PD-1/PD-L1) Interaction via Transiently Induced Protein States and Dimerization of PD-L1. J. Med. Chem. 2017, 60, 5857-5867. [CrossRef] [PubMed]

23. Acúrcio, R.C.; Scomparin, A.; Conniot, J.; Salvador, J.A.R.; Satchi-Fainaro, R.; Florindo, H.F.; Guedes, R.C. Structure-Function Analysis of Immune Checkpoint Receptors to Guide Emerging Anticancer Immunotherapy. J. Med. Chem. 2018, 61, 10957-10975. [CrossRef] [PubMed]

24. Huck, B.R.; Kötzner, L.; Urbahns, K. Small Molecules Drive Big Improvements in Immuno-Oncology Therapies. Angew. Chem. Int. Ed. 2018, 57, 4412-4428. [CrossRef] [PubMed]

25. Sasikumar, P.G.; Ramachandra, M. Small-Molecule Immune Checkpoint Inhibitors Targeting PD-1/PD-L1 and Other Emerging Checkpoint Pathways. BioDrugs 2018, 32, 481-497. [CrossRef] [PubMed]

26. Yang, J.; Hu, L. Immunomodulators targeting the PD-1/PD-L1 protein-protein interaction: From antibodies to small molecules. Med. Res. Rev. 2019, 39, 265-301. [CrossRef]

27. Zarganes-Tzitzikas, T.; Konstantinidou, M.; Gao, Y.; Krzemien, D.; Zak, K.; Dubin, G.; Holak, T.A.; Dömling, A. Inhibitors of programmed cell death 1 (PD-1): A patent review (2010-2015). Expert Opin. Ther. Pat. 2016, 26, 973-977. [CrossRef] [PubMed]

28. Konstantinidou, M.; Zarganes-Tzitzikas, T.; Magiera-Mularz, K.; Holak, T.A.; Dömling, A. Immune Checkpoint PD-1/PD-L1: Is There Life Beyond Antibodies? Angew. Chem. Int. Ed. 2018, 57, 4840-4848. [CrossRef]

29. Shaabani, S.; Huizinga, H.P.S.; Butera, R.; Kouchi, A.; Guzik, K.; Magiera-Mularz, K.; Holak, T.A.; Dömling, A. A patent review on PD-1/PD-L1 antagonists: Small molecules, peptides, and macrocycles (2015-2018). Expert Opin. Ther. Pat. 2018, 28, 665-678. [CrossRef]

30. Lazorchak, A.S.; Patterson, T.; Ding, Y.; Sasikumar, P.G.; Sudarshan, N.S.; Gowda, N.M.; Ramachandra, R.K.; Samiulla, D.S.; Giri, S.; Eswarappa, R.; et al. Abstract A36: CA-170, an oral small molecule PD-L1 and VISTA immune checkpoint antagonist, promotes $\mathrm{T}$ cell immune activation and inhibits tumor growth in pre-clinical models of cancer. In Proceedings of the AACR Special Conference on Tumor Immunology and Immunotherapy, Boston, MA, USA, 20-23 October 2016; p. A36.

31. Sasikumar, P.; Sudarshan, N.S.; Gowda, N.; Samiulla, D.S.; Ramachandra, R.; Chandrasekhar, T.; Adurthi, S.; Mani, J.; Nair, R.; Singh, S.; et al. Abstract 4861: Oral immune checkpoint antagonists targeting PD-L1/VISTA or PD-L1/Tim3 for cancer therapy. In Proceedings of the AACR 107th Annual Meeting 2016, New Orleans, LA, USA, 16-20 April 2016; p. 4861.

32. Available online: http://www.curis.com/images/stories/pdfs/posters/SITC2018CA-170RPD962.pdf (accessed on 5 June 2019).

33. Available online: http://www.curis.com/images/stories/pdfs/posters/SITC2018CA-170RPD961.pdf (accessed on 5 June 2019).

34. Available online: http://www.curis.com/images/stories/pdfs/posters/SITC2018CA-170P714ASIAD.pdf (accessed on 5 June 2019).

35. Sasikumar, P.G.; Ramachandra, R.K.; Adurthi, S.; Dhudashiya, A.A.; Vadlamani, S.; Vemula, K.; Vunnum, S.; Satyam, L.K.; Samiulla, D.S.; Subbarao, K.; et al. A Rationally Designed Peptide Antagonist of the PD-1 Signaling Pathway as an Immunomodulatory Agent for Cancer Therapy. Mol. Cancer Ther. 2019, 18, 1081-1091. [CrossRef]

36. Sasikumar, P.G.N.; Ramachandra, M.; Naremadde-palli, S.S.S. WO 2015/033301 Al 2015. Available online: http://www.eapatis.com/getdocument.asp?Document=//patstorage/EapatisStorage/wo/wo215010/ wo2015033301a1.pdf (accessed on 5 June 2019).

37. CA-170 MedKoo. Available online: https://medkoo.com/products/18283 (accessed on 5 June 2019).

38. CA-170 - InvivoChem. Available online: https://www.invivochem.com/ca-170/ (accessed on 5 June 2019).

39. CA-170 Glixxlabs. Available online: https://www.glixxlabs.com/chemical-products/bioactive-screen-leadsp6/GLXC-15291 (accessed on 5 June 2019).

40. CA-170. Available online: http://www.dcchemicals.com/product_show-PD_1_IN_1.html (accessed on 5 June 2019).

41. Sasikumar, P.G.N.; Ramachandra, M.; Vadlamani, S.K.; Vemula, K.R.; Satyam, L.K.; Subbarao, K.; Shrimali, K.R.; Kandepu, S. Immunosuppression Modulating Compounds. U.S. Patent 2011/0318373 A1 2011, 29 December 2011. 
42. Skalniak, L.; Zak, K.M.; Guzik, K.; Magiera, K.; Musielak, B.; Pachota, M.; Szelazek, B.; Kocik, J.; Grudnik, P.; Tomala, M.; et al. Small-molecule inhibitors of PD-1/PD-L1 immune checkpoint alleviate the PD-L1-induced exhaustion of T-cells. Oncotarget 2017, 8, 72167-72181. [CrossRef]

43. Rehm, T.; Huber, R.; Holak, T.A. Application of NMR in Structural Proteomics. Structure 2002, 10, $1613-1618$. [CrossRef]

44. Shuker, S.B.; Hajduk, P.J.; Meadows, R.P.; Fesik, S.W. Discovering High-Affinity Ligands for Proteins: SAR by NMR. Science 1996, 274, 1531-1534. [CrossRef] [PubMed]

45. Degorce, F. HTRF: A Technology Tailored for Drug Discovery-A Review of Theoretical Aspects and Recent Applications. Curr. Chem. Genom. 2009, 3, 22-32. [CrossRef] [PubMed]

46. Cheng, Z.J.; Karassina, N.; Grailer, J.; Hartnett, J.; Fan, F.; Cong, M. Abstract 5440: Novel PD-1 blockade bioassay to assess therapeutic antibodies in PD-1 and PD-L1 immunotherapy programs. In Proceedings of the AACR 106th Annual Meeting 2015, Philadelphia, PA, USA, 18-22 April 2015; p. 5440.

47. Wang, C.; Thudium, K.B.; Han, M.; Wang, X.T.; Huang, H.; Feingersh, D.; Garcia, C.; Wu, Y.; Kuhne, M.; Srinivasan, M.; et al. In vitro characterization of the anti-PD-1 antibody nivolumab, BMS-936558, and in vivo toxicology in non-human primates. Cancer Immunol. Res. 2014, 2, 846-856. [CrossRef] [PubMed]

48. Blevins, D.; Hanley, R.; Bolduc, T.A.; Powell, D.; Gignac, M.; Walker, K.D.; Carr, M.; Hof, F.E.; Wulff, J. In Vitro Assessment of Putative PD-1/PD-L1 Inhibitors: Suggestions of an Alternative Mode of Action. ACS Med. Chem. Lett. 2019. [CrossRef]

49. Magiera-Mularz, K.; Skalniak, L.; Zak, K.M.; Musielak, B.; Rudzinska-Szostak, E.; Berlicki, Ł.; Kocik, J.; Grudnik, P.; Sala, D.; Zarganes-Tzitzikas, T.; et al. Bioactive Macrocyclic Inhibitors of the PD-1/PD-L1 Immune Checkpoint. Angew. Chem. Int. Ed. 2017, 56, 13732-13735. [CrossRef]

50. Van Kuppeveld, F.J.M.; Van der Logt, J.T.M.; Angulo, A.F.; Van Zoest, M.J.; Quint, W.G.V.; Niesters, H.G.M.; Galama, J.M.D.; Melchers, W.J.G. Genus- and species-specific identification of mycoplasmas by 16S rRNA amplification. Appl. Environ. Microbiol. 1992, 58, 2606-2615. [PubMed]

Sample Availability: Samples of the compounds are not available.

(C) 2019 by the authors. Licensee MDPI, Basel, Switzerland. This article is an open access article distributed under the terms and conditions of the Creative Commons Attribution (CC BY) license (http://creativecommons.org/licenses/by/4.0/). 